\title{
Examen du test de dépistage rapide du virus de l'immunodéficience humaine (VIH)
}

\author{
Ha $\mathbf{S}^{1^{*}}$, Foley $\mathrm{S}^{1}$, Paquette $\mathrm{D}^{1}$, Seto $\mathrm{J}^{1}$
}

${ }^{1}$ Centre de la lutte contre les maladies transmissibles et les infections, Agence de la santé publique du Canada, Ottawa (Ontario)

*Correspondance: shalane.ha@phac-aspc.gc.ca

\section{Résumé}

Contexte : Au Canada, on estime que 71300 personnes vivaient avec le VIH à la fin de 2011. Environ $25 \%$ (14 500 à 21500 ) des cas prévalents ignoraient qu'ils étaient infectés par le VIH. Une utilisation élargie du dépistage rapide du VIH pourrait augmenter la détection d'infections non diagnostiquées, permettre un traitement et des services de soutien plus précoces et prévenir la retransmission soutenue du virus.

Objectif : Examiner l'acceptabilité par les patients, l'incidence (définie comme la réception des résultats et le lien aux soins) et le rapport coût-efficacité du dépistage rapide du VIH.

Méthodologie : Une recherche d'examens systématique du dépistage rapide du VIH a été menée, à partir d'études menées dans des pays en développement et dans des pays développés du monde entier, publiés en anglais, entre 2000 et 2013. L'outil AMSTAR (évaluation méthodologique de la qualité des examens systématiques) a été utilisé pour évaluer la qualité méthodologique des examens systématiques inclus. Les résultats ont été résumés de façon descriptive pour chacun des résultats.

Résultats : Huit examens systématiques ont été inclus. L'acceptabilité du dépistage rapide du VIH était généralement élevée dans des contextes médicaux ( $69 \%$ à $98 \%$ ), spécialement chez les femmes enceintes et les jeunes se présentant dans des salles d'urgence, mais était inférieure dans des contextes non médicaux (14\% à $46 \%$ ). Le pourcentage de personnes ayant obtenu le résultat de leur test était variable. II était élevé ( $83 \%$ à $93 \%$ ) dans les salles d'urgence mais était faible en contexte de soins accélérés à heures normales de travail (27\%). L'incidence sur la collaboration aux soins était limitée. Un seul examen systématique a porté sur le rapport coût-efficacité du dépistage rapide et a permis de conclure que le dépistage rapide du VIH était rentable, en comparaison avec les méthodes traditionnelles; toutefois, les résultats étaient tous fondés sur des modèles statiques.

Conclusion : Dans l'ensemble, le dépistage rapide du VIH a révélé un niveau élevé d'acceptabilité, une variabilité dans la réception des résultats de tests et une incidence limitée sur la collaboration aux soins. Bien que ces constatations suggèrent que le dépistage rapide du VIH peut être utile, des recherches plus approfondies sont requises pour confirmer chez qui, quand et où ils sont le mieux utilisés, ainsi que la façon d'améliorer la collaboration aux soins.

\section{Introduction}

À la fin de 2011, on estime que 71300 personnes vivaient avec le virus de l'immunodéficience humaine (VIH) et le syndrome de l'immunodéficience acquise (sida) au Canada et environ $25 \%$ ignoraient leur sérologie $\mathrm{VIH}$ (1). Les personnes ignorant leur sérologie VIH sont incapables de tirer parti des services de soutien et des soins, présentent un risque accru de transmission du VIH ainsi que de contracter d'autres infections transmissibles sexuellement et par le sang. Des stratégies efficaces de dépistage permettant le diagnostic et le traitement précoces peuvent contribuer à améliorer les résultats pour la santé individuelle et de la population (2).

Avec l'émergence de nouvelles technologies diagnostiques, le nombre d'options de tests du VIH augmente. Des tests rapides de dépistage du VIH sont disponibles à l'échelle mondiale, y compris sous forme de tests de fluide buccal et de tests de sang entier ou de plasma prélevé par piqûre au bout du doigt. Les tests de dépistage rapide du VIH peuvent se 
faire par autoadministration ou peuvent être administrés par un personnel formé. Au Canada, les tests de dépistage rapide du VIH peuvent uniquement être effectués par un personnel formé, en contexte de point de service (p. ex. : cabinet de médecin, clinique, services d'urgence) (3-5). En outre, l'Agence de la santé publique du Canada recommande que les tests de dépistage rapide du VIH soient administrés avec du counseling prétest et post-test (5).

Un seul test de dépistage rapide du VIH est homologué pour utilisation au Canada (6). En octobre 2005, Santé Canada a approuvé le test INSTI de détection des anticorps anti-VIH-1 (un test de dépistage rapide du VIH à une seule utilisation) devant être utilisé en contexte de point service. En 2008, le permis a été modifié afin d'inclure le test INSTI de détection des anticorps anti-VIH-1/VIH-2 (6). II s'agit d'un test de détection des anticorps préliminaire pouvant être administré sur les lieux, où le patient peut recevoir les résultats immédiatement $(<1 \mathrm{~h})(7-10)$. Si un patient reçoit un résultat de test rapide réactif, un test de confirmation traditionnel en laboratoire est requis. Si le résultat du test est négatif (non réactif), aucun test supplémentaire n'est requis $(3,5)$.

Des études précédentes suggèrent que les tests en contexte de point de service ont le potentiel d'améliorer la gestion des maladies infectieuses en détectant de nouvelles infections, réduisant ainsi le nombre de personnes ignorant qu'elles sont infectées et facilitant la collaboration aux soins $(11,12)$. Pour s'assurer que les tests de dépistage rapide du VIH sont possibles, ils doivent également être rentables. L'objectif de cette revue rapide est d'examiner les données les plus actuelles sur l'acceptabilité par les patients, l'incidence (définie comme la réception des résultats de tests et la collaboration aux soins) et la rentabilité des tests de dépistage rapide du VIH.

\section{Méthodologie}

Nous avons suivi les méthodes de l'Institut de recherche de l'Hôpital d'Ottawa pour effectuer des revues rapides (13). Cette méthode est conçue afin de fournir rapidement aux décideurs une synthèse d'une riche documentation (13). Un protocole de revue rapide a priori a été élaboré, comprenant : l'élaboration et l'amélioration des questions; une recherche systématique de la littérature; la présélection et la sélection de revues systématiques; l'évaluation de la qualité des données; une synthèse descriptive des études incluses. (13).

\section{Stratégie de recherche}

Les bases de données suivantes ont été interrogées : Medline, Embase, Scopus, Social Policy and Practice, Proquest Public Health et Google Scholar. Les articles ont été inclus s'ils ont été publiés entre janvier 2000 et septembre 2013; les études provenant de pays développés et de pays en développement ont été incluses; les études sélectionnées étaient publiées en anglais. La stratégie de recherche incluait les mots-clés suivants : ( human immunodeficiency virus » OU " VIH ») ET ( " Point of care » OU « point-of-care », " rapid test » OU « home-based test » OU « screen* ») ET (« linkage to care » OU « follow-up » OU « barrier* », « intervention* » OU « access* » OU « diagnos* ») OU («acceptab* »,

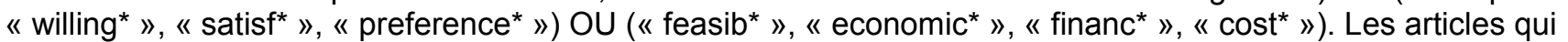
communiquaient des résultats sur la prévalence du $\mathrm{VIH}$ ou les études ne faisant pas mention des tests de dépistage rapide du VIH ont été exclus de la revue.

\section{Évaluation de la qualité des études}

La qualité de la méthodologie de chaque examen systématique a été évaluée à l'aide de l'outil AMSTAR (14). L'outil AMSTAR consiste en un questionnaire à 11 éléments évaluant les critères suivants : utilisation d'une méthodologie a priori; sélection d'études en double et processus d'extraction de données; recherche approfondie de la littérature; utilisation du statut des publications comme critère d'inclusion; caractéristiques des études incluses; liste d'études incluses/exclues; évaluation de la qualité des études; utilisation appropriée du jugement scientifique dans la formulation de conclusions; utilisation de méthodes appropriées pour combiner les constatations des études; évaluation de la possibilité de biais de publication; reconnaissance de conflit d'intérêts. Pour s'assurer de la fiabilité de l'évaluation, deux des auteurs (SH, SF) ont évalué les examens systématiques à l'aide de l'outil AMSTAR. En cas d'irrégularités, une tierce partie a été invitée à évaluer le critère en question (DP).

\section{Extraction des données}

Pour chacun des examens systématiques inclus, deux auteurs (SH, SF) ont extrait des données sur la population; effectué des recherches en fonction des années, du nombre d'études incluses, de l'emplacement des études incluses, des objectifs des études, du type d'intervention et des résultats. Les résultats présentant un intérêt comprenaient : l'acceptabilité, la réception des résultats de tests de dépistage du $\mathrm{VIH}$, la collaboration aux soins et la rentabilité. Après l'extraction de données, les deux auteurs ont comparé leurs constatations pour veiller à l'uniformité. 


\section{Résultats}

La recherche initiale a permis d'obtenir 892 articles sur le dépistage rapide du VIH. Après avoir limité la sélection aux examens systématiques $(n=12)$, chaque article de la revue a respecté les critères d'inclusion (figure 1).

Figure 1 : Algorithme de recherche de la littérature et sélection d'examens systématiques sur le dépistage rapide du VIH dans le cadre de l'étude

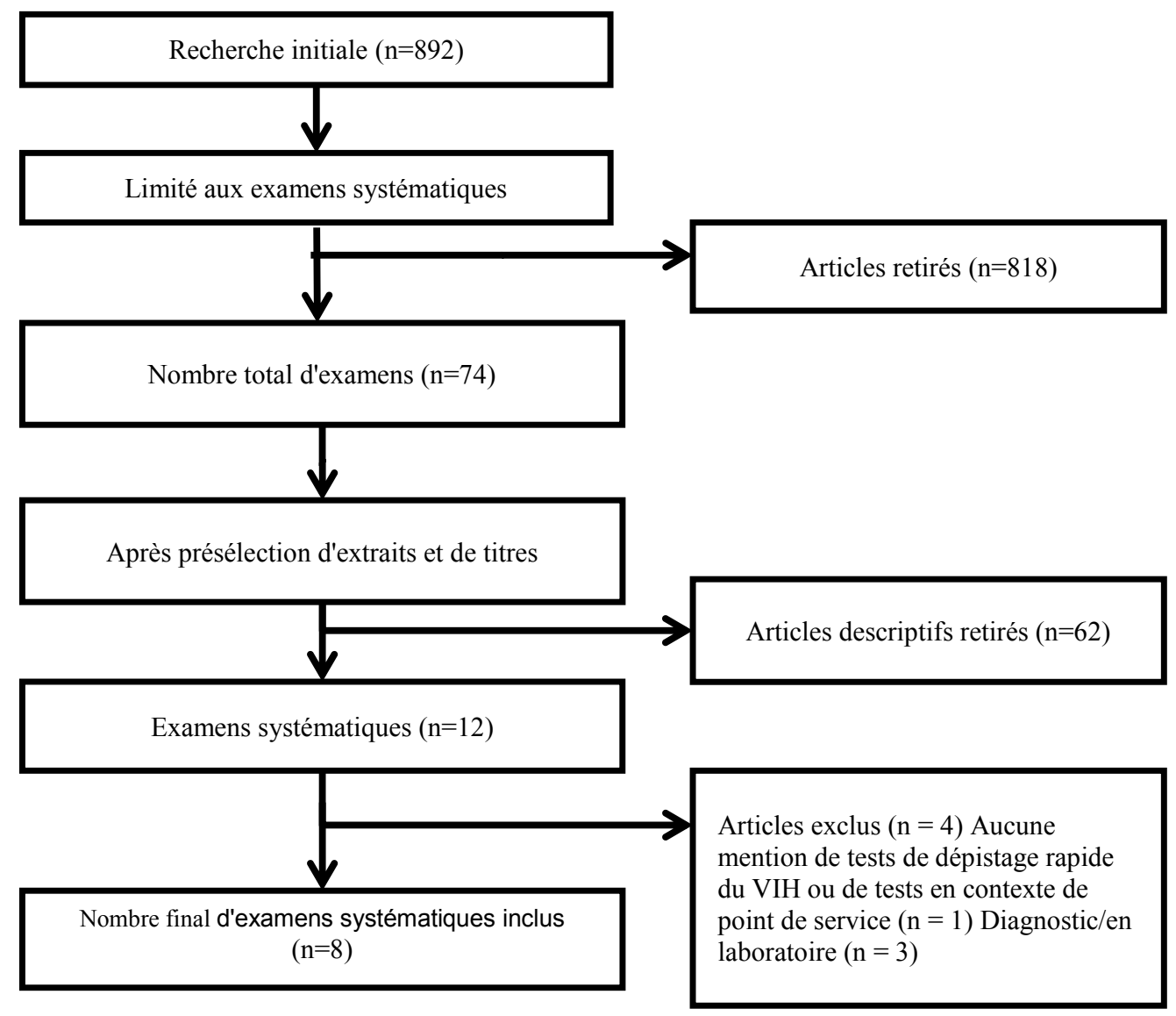

Une description des examens inclus et leur score respectif à l'outil AMSTAR sur 11 figure au tableau 1. Trois ont obtenu un score parfait à l'outil AMSTAR et un autre était de qualité élevée (avec un score de 8). Parmi les raisons pour lesquelles certains examens systématiques ont obtenu un score inférieur à huit, on compte : absence de sélection d'études en double et processus d'extraction de données; évaluation et documentation de la qualité des études; évaluation de biais de publication. 
Tableau 1 : Description des examens systématiques inclus avec les scores AMSTAR ${ }^{1}$.

\begin{tabular}{|c|c|c|c|c|}
\hline Référence & Objectifs & $\begin{array}{l}\text { Population et } \\
\text { emplacement }\end{array}$ & $\begin{array}{l}\text { Période de recherche, } \\
\text { intervention et nombre } \\
\text { d'études incluses }\end{array}$ & $\begin{array}{l}\text { Score } \\
\text { AMSTAR } \\
\text { (sur 11) }\end{array}$ \\
\hline Bateganya (2007) (17) & $\begin{array}{l}\text { Trouver et évaluer de manière } \\
\text { critique les études portant sur la } \\
\text { mise en œuvre de counseling et } \\
\text { de dépistage volontaire à } \\
\text { domicile du VIH; évaluer l'effet } \\
\text { de cette intervention, en } \\
\text { comparaison avec le counseling } \\
\text { et le dépistage du VIH en } \\
\text { établissement. }\end{array}$ & $\begin{array}{l}\text { Population : Adultes } \\
\text { (> } 15 \text { ans) } \\
\text { Emplacements : } \\
\text { Uganda et Zambie }\end{array}$ & $\begin{array}{l}\text { Période visée par la } \\
\text { recherche : 1980-2007 } \\
\text { Intervention : counseling } \\
\text { et dépistage du VIH } \\
\text { volontaire } \\
\text { Nombre d'études } \\
\text { incluses : } 2\end{array}$ & 11 \\
\hline Bateganya (2010) (8) & $\begin{array}{l}\text { Établir l'effet du counseling et du } \\
\text { dépistage volontaire à domicile } \\
\text { du VIH sur l'adoption du } \\
\text { dépistage du VIH. }\end{array}$ & $\begin{array}{l}\text { Population : Adultes } \\
\text { (> } 15 \text { ans) } \\
\text { Emplacements : } \\
\text { Zambie }\end{array}$ & $\begin{array}{l}\text { Période visée par la } \\
\text { recherche : } 2007-2008 \\
\text { Intervention : counseling } \\
\text { et dépistage du VIH } \\
\text { volontaire } \\
\text { Nombre d'études } \\
\text { incluses : } 1^{1}\end{array}$ & 11 \\
\hline $\begin{array}{l}\text { Dibosa-Osadolor } \\
(2010)(21)\end{array}$ & $\begin{array}{l}\text { Examiner les données utilisées } \\
\text { pour estimer la rentabilité du } \\
\text { dépistage du VIH et évaluer les } \\
\text { méthodologies des études } \\
\text { économiques portant sur le } \\
\text { dépistage du VIH. }\end{array}$ & $\begin{array}{l}\text { Populations : Diverses } \\
\text { Emplacement : Non } \\
\text { précisé }\end{array}$ & $\begin{array}{l}\text { Période visée par la } \\
\text { recherche : 1993-2008 } \\
\text { Intervention : } \\
\text { Modélisation économique } \\
\text { des programmes de } \\
\text { dépistage et de diagnostic } \\
\text { du VIH } \\
\text { Nombre d'études } \\
\text { incluses : } 17\end{array}$ & 7 \\
\hline $\begin{array}{l}\text { Napierala Mavedzenge } \\
(2013)(18)\end{array}$ & $\begin{array}{l}\text { Mener un examen des politiques } \\
\text { et de la recherche sur } \\
\text { l'autoadministration de tests de } \\
\text { dépistage du VIH. }\end{array}$ & $\begin{array}{l}\text { Populations : Diverses } \\
\text { Emplacements : } \\
\text { Kenya, Zambie, États- } \\
\text { Unis, Singapour, Afrique } \\
\text { du Sud, Allemagne, } \\
\text { Malawi, Pays-Bas, } \\
\text { Royaume-Uni, France }\end{array}$ & $\begin{array}{l}\text { Période visée par la } \\
\text { recherche : } 1980 \text { - } \\
\text { mai } 2012 \\
\text { Intervention : } \\
\text { Autoadministration de } \\
\text { tests de dépistage du VIH } \\
\text { Nombre d'études } \\
\text { incluses : } 24\end{array}$ & 6 \\
\hline Pant Pai (2007) (15) & $\begin{array}{l}\text { Résumer la précision globale } \\
\text { des tests de diagnostic rapide } \\
\text { du VIH pendant la grossesse; } \\
\text { évaluer les résultats et } \\
\text { l'incidence des tests; déterminer } \\
\text { les obstacles pratiques liés à la } \\
\text { mise en œuvre de } \\
\text { l'autoadministration de tests de } \\
\text { dépistage du VIH et du } \\
\text { counseling chez les femmes } \\
\text { enceintes. }\end{array}$ & $\begin{array}{l}\text { Population : Femmes } \\
\text { enceintes (18 à } 44 \text { ans) } \\
\\
\text { Emplacements : } \\
\text { Afrique du Sud, États- } \\
\text { Unis, Amérique latine, } \\
\text { Asie du Sud-Est, } \\
\text { Jamaïque }\end{array}$ & $\begin{array}{l}\text { Période visée par la } \\
\text { recherche : } 1991 \text { - juillet } \\
2005 \\
\text { Intervention : Tests de } \\
\text { dépistage du VIH en } \\
\text { contexte de point de } \\
\text { service chez les femmes } \\
\text { enceintes. } \\
\text { Nombre d'études } \\
\text { incluses : } 17\end{array}$ & 8 \\
\hline Pant Pai (2013) (9) & $\begin{array}{l}\text { Examiner les stratégies } \\
\text { d'autoadministration supervisée } \\
\text { et non supervisée de tests de } \\
\text { dépistage du VIH. }\end{array}$ & $\begin{array}{l}\text { Populations : Diverses } \\
\text { Emplacements : États- } \\
\text { Unis, Canada, } \\
\text { Singapour, Inde, Malawi, } \\
\text { Espagne, Kenya, Pays- } \\
\text { Bas }\end{array}$ & $\begin{array}{l}\text { Période visée par la } \\
\text { recherche : janvier } 2000 \text { - } \\
\text { octobre } 2012 \\
\text { Intervention : } \\
\text { Administration supervisée } \\
\text { et non supervisée de tests } \\
\text { de dépistage du VIH en }\end{array}$ & 11 \\
\hline
\end{tabular}




\begin{tabular}{|c|c|c|c|c|}
\hline & & & $\begin{array}{l}\text { contexte de point de } \\
\text { service. } \\
\text { Nombre d'études } \\
\text { incluses : } 21\end{array}$ & \\
\hline Roberts (2007) (16) & $\begin{array}{l}\text { Examiner les résultats des tests } \\
\text { rapides de dépistage du VIH } \\
\text { sous forme de tests de fluide } \\
\text { buccal et de tests de sang. }\end{array}$ & $\begin{array}{l}\text { Populations : Diverses } \\
\text { Emplacements : États- } \\
\text { Unis, Kenya, Brésil, } \\
\text { Zimbabwe, Burkina } \\
\text { Faso, Mexique }\end{array}$ & $\begin{array}{l}\text { Période visée par la } \\
\text { recherche : janvier } 2000 \text { - } \\
\text { juin } 2006 \\
\text { Intervention : Dépistage } \\
\text { rapide du VIH. } \\
\text { Nombre d'études } \\
\text { incluses : } 26\end{array}$ & 4 \\
\hline Turner (2013) (19) & $\begin{array}{l}\text { Examiner les préférences et } \\
\text { l'acceptabilité du dépistage en } \\
\text { contexte de point de service } \\
\text { chez les jeunes, documenter les } \\
\text { taux de notification et déterminer } \\
\text { les facteurs sociaux et } \\
\text { démographiques associés à la } \\
\text { préférence des jeunes pour les } \\
\text { tests de dépistage rapide en } \\
\text { contexte de point de service } \\
\text { plutôt que les tests traditionnels. }\end{array}$ & $\begin{array}{l}\text { Population : Les } \\
\text { jeunes. } \\
(<25 \text { ans }) \\
\text { Emplacement : États- } \\
\text { Unis }\end{array}$ & $\begin{array}{l}\text { Période visée par la } \\
\text { recherche : janvier } 1990 \text { - } \\
\text { mars } 2013 \\
\text { Intervention : Tests de } \\
\text { dépistage du VIH en } \\
\text { contexte de point de } \\
\text { service. } \\
\text { Nombre d'études } \\
\text { incluses : } 14\end{array}$ & 7 \\
\hline
\end{tabular}

${ }^{1} \mathrm{AMSTAR}=$ évaluation méthodologique de la qualité des examens systématiques. Il s'agit d'un outil utilisé pour évaluer la qualité méthodologique des examens systématiques.

${ }^{2}$ Cet examen a inclus une étude, car il s'agissait d'une mise à jour de l'examen de Bateganya (2007) Cochrane.

\section{Acceptabilité}

Presque tous les examens (7/8) ont étudié l'acceptabilité. L'acceptabilité a été définie dans ces examens comme l'adoption par la population des tests de dépistage rapide $(8,9,15-17)$ ou comme la préférence pour le patient d'un test de dépistage rapide, lorsqu'on lui offre le choix, par rapport au test en laboratoires traditionnel $(18,19)$.

Dans l'étude de Roberts et al., l'acceptabilité globale des tests de dépistage rapide administrés en contexte médical et communautaire a varié de $14 \%$ à $98 \%$ (16). L'acceptabilité du dépistage rapide était inférieure (14\% à $46 \%)$ dans les sites de test non traditionnels (p. ex. : bains publics, programmes d'échange de seringues, prisons et services d'urgence), par rapport au contexte médical $(69 \%$ à $98 \%$ ) (p. ex. : cliniques pour infections transmises sexuellement, unités réservées à l'accouchement et hôpitaux) (16). L'important écart dans les taux d'acceptation peut avoir été influencé par des différences dans la définition de l'acceptabilité et par les méthodes de collecte de données.

Dans deux examens, l'acceptabilité des tests de dépistage rapide du VIH a été élevée chez les femmes enceintes $(15,16)$. Dans l'examen mené par Pant Pai et al., l'acceptabilité chez les femmes enceintes a été de $83 \%$ à $97 \%(15)$. De façon similaire, dans l'examen de Roberts et al., l'acceptabilité chez les femmes enceintes a été de $74 \%$ à $86 \%$ dans les études américaines et de $93 \%$ à $98 \%$ dans les études internationales (16). Chez les femmes enceintes, les facteurs suivants ont été associés à un niveau élevé d'acceptabilité des tests de dépistage rapide du VIH : âge (<21 ans), niveau d'éducation supérieur et manque de soins prénataux appropriés pendant la grossesse (15).

Chez les jeunes, l'examen de Turner et al. révèle que $35 \%$ à $93 \%$ des sujets ont accepté le test de dépistage rapide du VIH lorsque celui-ci leur était offert. Le taux d'acceptation de $35 \%$ a été constaté dans une clinique externe pour adolescents (19). Toutefois, en présence du choix entre les méthodes rapides ou les méthodes traditionnelles, les adolescents de cliniques externes ont sélectionné les méthodes rapides dans $70 \%$ des cas (19). Les taux d'acceptation les plus élevés ( $83 \%$ à $93 \%$ ) ont été observés dans des salles d'urgence, ce qui porte à croire qu'il y a un taux d'acceptation élevé des tests rapides chez les jeunes se présentant aux services d'urgence (19).

Dans l'examen de Mavedzenge et al., l'acceptabilité a été définie comme l'intérêt à l'égard de l'autoadministration. Chez les populations clés comme celle des hommes ayant des relations sexuelles avec d'autres hommes (HARSAH) et les personnes se présentant aux services d'urgence, les auteurs ont constaté que l'acceptabilité à l'égard de l'autoadministration était de moyenne à élevée (62\% à $92 \%)$ (18). Les raisons liées à la préférence pour l'autoadministration comprenaient la vie privée, l'autonomie, la confidentialité, l'anonymat, la commodité et la rapidité. 
Pant Pai et al. ont démontré que l'acceptabilité (choisir l'autoadministration plutôt que les méthodes de tests en laboratoire traditionnelles) était élevée en contexte supervisé et non supervisé (9). En contexte supervisé, l'acceptabilité était élevée (74 \% à $96 \%$ ) chez les personnes se présentant dans les services d'urgence, les HARSAH en contexte urbain, les étudiants universitaires et la population urbaine générale. II convient de mentionner qu'une étude datant de 2001 a fait état d'un taux d'acceptation de $24 \%$ chez les personnes fréquentant une clinique de soins liés au VIH. En contexte non supervisé, l'acceptabilité élevée (74 \% à $84 \%)$ était fondée sur deux études seulement, qui mettaient l'accent sur les professionnels de la santé et les HARSAH séronégatif pour le VIH (9).

L'acceptabilité du dépistage rapide du VIH était variable parmi les différentes populations, mais était généralement élevée chez les femmes enceintes, les jeunes se présentant aux services d'urgence et en contexte médical. Des recherches plus approfondies sont requises afin d'explorer l'autoadministration en contexte non supervisé et les raisons des faibles taux d'acceptation en contexte non médical.

\section{Réception des résultats de tests de dépistage du VIH}

Quatre des huit (4/8) examens systématiques ont étudié l'incidence des tests de dépistage rapide du VIH sur la réception des résultats par les patients. Un examen systématique mené par Roberts et al. a permis de constater que $27 \%$ à $100 \%$ des clients se présentant en contexte médical et communautaire afin de recevoir un test de dépistage rapide ont reçu les résultats de leur test (16). Le faible taux de $27 \%$ est attribuable au fait que des résultats étaient disponibles le jour même dans une clinique de soins d'urgence aux heures ouvrables normales et que la plupart des participants sont partis avant que les résultats ne soient disponibles (20). Dans les autres études, plus de $70 \%$ des participants auxquels on a administré des tests de dépistage rapide dans les hôpitaux, les cliniques de soins pour infections transmises sexuellement, les refuges pour sans-abri, et les bains publics ont reçu les résultats de leur test (16).

Dans un examen mené par Bateganya et al., les personnes ayant reçu du counseling et du dépistage volontaire (avec des tests rapides) à domicile étaient environ cinq fois plus susceptibles de recevoir les résultats de leur test, comparativement aux personnes ayant reçu du counseling et du dépistage volontaire dans une clinique (17). Les auteurs ont mené un examen mis à jour qui incluait une étude supplémentaire et ont déterminé que $56 \%$ des personnes ayant effectué leur test à domicile ont obtenu leurs résultats, comparativement à $12 \%$, pour ceux ayant effectué leur test en clinique (8). Selon ces constatations, la réception des résultats de tests de dépistage rapide avait tendance à être de modérée à élevée, sauf dans les cliniques de soins d'urgence aux heures ouvrables normales.

\section{Collaboration aux soins}

Six sur huit (6/8) examens systématiques ont évalué la collaboration aux soins, bien que la définition de la collaboration aux soins varie d'un examen à l'autre. Roberts et al. ont défini la collaboration aux soins comme l'administration de soins médicaux et a déterminé que cela se produisait dans $47 \%$ à $100 \%$ des cas recevant un diagnostic de $\mathrm{VIH}$ par test de dépistage rapide (16). Mavedzenge et al. ont défini la collaboration aux soins comme le lien avec des services de prévention, de traitement et de soins et ont conclu que les données sont insuffisantes pour déterminer si l'autoadministration de tests mène à une collaboration aux soins rapide (18). Dibosa-Osadolor et al. ont déterminé que les tests de dépistage rapide du VIH permettent un pourcentage plus élevé de patients pour lesquels la collaboration aux soins est appropriée, comparativement aux tests traditionnels de dépistage du VIH (21); toutefois, les pourcentages exacts n'étaient pas fournis. Bateganya et al. n'ont pas fourni de définition claire de la collaboration aux soins, mais ont inclus des études qui offraient du counseling volontaire prétest et post-test à domicile. En comparaison avec les personnes effectuant leur test et recevant du counseling en clinique, les personnes ayant reçu ces services à domicile étaient plus susceptibles d'accepter du counseling post-test (17). Dans l'examen mis à jour de Bateganya et al., $12 \%$ ont reçu du counseling post-test d'une clinique et $56 \%$, à domicile(8). La plupart des examens reconnaissent que l'information au sujet de la collaboration aux soins est insuffisante $(9,15,16)$.

Voir le tableau 2 pour obtenir un sommaire des données en matière d'acceptabilité, de réception des résultats de tests, et de collaboration aux soins. 
Tableau 2 : sommaire des données d'acceptabilité, de réception des résultats de tests et de collaboration aux soins par les références principales

\begin{tabular}{|c|c|c|c|}
\hline Référence & Acceptabilité & $\begin{array}{l}\text { Réception des résultats de tests } \\
\text { de dépistage du VIH }\end{array}$ & Collaboration aux soins \\
\hline $\begin{array}{l}\text { Bateganya } \\
(2007)(17)\end{array}$ & $\begin{array}{l}\text { Les personnes réparties aléatoirement } \\
\text { effectuant leur test dans des } \\
\text { emplacements optionnels (y compris les } \\
\text { tests à domicile) étaient } 4,6 \text { fois plus } \\
\text { susceptibles d'accepter du counseling et } \\
\text { le dépistage volontaire que les personnes } \\
\text { appartenant au groupe recevant ces } \\
\text { services en établissement (risque relatif } \\
\text { de } 4,6 \text {; IC à } 95 \% 3,6-6,2 \text { ) (26). }\end{array}$ & $\begin{array}{l}\text { Au cours de l'année où les } \\
\text { participants se sont vus donner le } \\
\text { choix de recevoir les résultats de } \\
\text { leur test de dépistage du VIH à la } \\
\text { maison, les ils étaient } 5,23 \text { fois plus } \\
\text { susceptibles de recevoir leurs } \\
\text { résultats qu'au cours de l'année où } \\
\text { les résultats étaient disponibles } \\
\text { seulement en établissement (rapport } \\
\text { de cotes de } 5,23 \text {; IC à } 95 \% 4,02- \\
6,8)(27) \text {. }\end{array}$ & $\begin{array}{l}\text { La définition de collaboration } \\
\text { aux soins n'était pas claire. II } \\
\text { semble que les personnes } \\
\text { ayant reçu leurs résultats ont } \\
\text { également reçu du } \\
\text { counseling post-test. }\end{array}$ \\
\hline $\begin{array}{l}\text { Bateganya } \\
(2010)(8)\end{array}$ & $\begin{array}{l}\text { L'acceptabilité du counseling prétest et du } \\
\text { test de dépistage du VIH était de } 12 \% \text { c. } \\
57 \% \text { (groupe disposant de l'option) (26). }\end{array}$ & $\begin{array}{l}12 \% \text { ont reçu du counseling post- } \\
\text { test et les résultats de leur test de la } \\
\text { clinique locale; } 56 \% \text { ont reçu leurs } \\
\text { résultats et du counseling à domicile } \\
\text { (risque relatif de } 4,7 ; \text { IC à } 95 \% 3,62- \\
6,21)(26) \text {. }\end{array}$ & $\begin{array}{l}\text { La définition de collaboration } \\
\text { aux soins n'était pas claire. II } \\
\text { semble que les personnes } \\
\text { ayant reçu leurs résultats ont } \\
\text { également reçu du } \\
\text { counseling post-test. }\end{array}$ \\
\hline $\begin{array}{l}\text { Dibosa-Osadolor } \\
(2010)(21)\end{array}$ & S.o. & s.o. & $\begin{array}{l}\text { Le dépistage rapide des } \\
\text { anticorps a aussi donné lieu } \\
\text { à un pourcentage plus élevé } \\
\text { de patients pour lesquels la } \\
\text { collaboration aux soins était } \\
\text { appropriée (28-31). }\end{array}$ \\
\hline $\begin{array}{l}\text { Napierala } \\
\text { Mavedzenge } \\
(2013)(18)\end{array}$ & $\begin{array}{l}\text { Les travailleurs de la santé de pays } \\
\text { africains ont manifesté un vif intérêt pour } \\
\text { l'autoadministration de tests, à savoir } \\
\text { dans } 73 \% \text { à } 79 \% \text { des cas (32-34). } \\
\text { Dans des études menées aux États-Unis, } \\
\text { des patients de services d'urgence et des } \\
\text { HARSAH }{ }^{2} \text { ont présenté un niveau élevé } \\
\text { d'acceptabilité, de l'ordre de } 83 \% \text { à } 89 \% \\
\text { (35-37). }\end{array}$ & s.o. & Données insuffisantes. \\
\hline $\begin{array}{l}\text { Pant Pai (2007) } \\
\text { (15) }\end{array}$ & $\begin{array}{l}\text { Acceptabilité globale : } 83 \% \text { à } 97 \% \text { (38- } \\
\text { 42). } \\
\text { II n'y a aucun consensus clair pour ce qui } \\
\text { est de la préférence des patients pour la } \\
\text { méthode de test rapide (p. ex. : sanguin } \\
\text { ou fluide buccal). }\end{array}$ & s.o. & $\begin{array}{l}\text { Les détails sur la } \\
\text { collaboration aux soins et la } \\
\text { prévention n'ont pas été } \\
\text { déclarés. }\end{array}$ \\
\hline $\begin{array}{l}\text { Pant Pai (2013) } \\
\text { (9) }\end{array}$ & $\begin{array}{l}\text { Acceptabilité globale : } 74 \% \text { à } 96 \% \text { en } \\
\text { contexte supervisé et non supervisé } \\
(7,35,43-49) \text {. } \\
\text { Contexte supervisé : } 24 \% \text { à } 95 \% \\
(7,35,43-47) \\
\text { - } \quad \text { HARSAH en contexte urbain : } 74 \% \\
(35) \\
\text { - } \quad \text { Services d'urgence : } 85 \% \text { ( } 7) \\
\text { - } \quad \text { Site de test de dépistage rapide du } \\
\text { VIH : } 78 \% \text { (45) } \\
\text { - Population urbaine générale : } 92 \% \\
\text { (46,47) } \\
\text { - Étudiants éduqués : } 95 \%(44) \\
\text { - Personnes fréquentant une clinique } \\
\text { de soins pour l'infection au VIH : } \\
24 \% \text { (43) } \\
\text { Contexte non supervisé : } 78 \% \text { à } 84 \% \\
\text { (48,49) } \\
\text { - HARSAH non monogames : } 84 \% \\
\text { (49) } \\
\text { - Professionnels de la santé : } 78 \% \\
\text { (48) }\end{array}$ & s.o. & $\begin{array}{l}\text { Une seule étude en contexte } \\
\text { non supervisé aux États- } \\
\text { Unis a été déclarée : } \rightarrow \\
96 \% \text { des personnes } \\
\text { séropositives au VIH } \\
\text { solliciteraient du counseling } \\
\text { post-test (50). }\end{array}$ \\
\hline
\end{tabular}




\begin{tabular}{|c|c|c|c|}
\hline $\begin{array}{l}\text { Roberts (2007) } \\
(16)\end{array}$ & 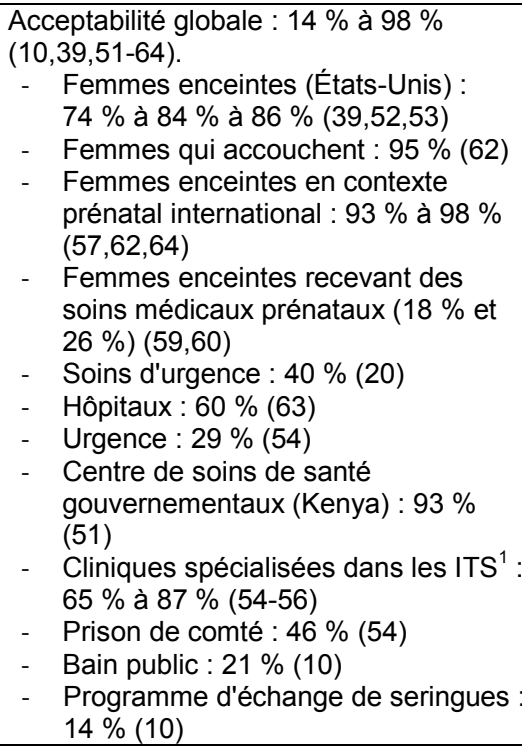 & 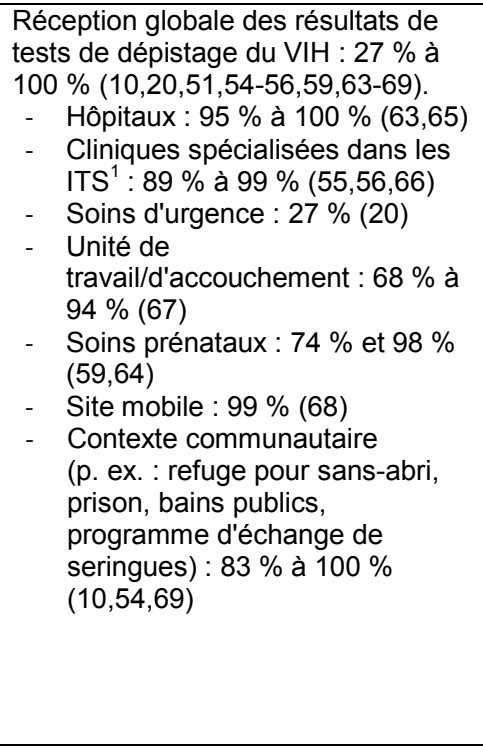 & $\begin{array}{l}\text { Global : } 47 \% \text { à } 100 \% \\
\text { (toutes les études pour les } \\
\text { États-Unis) }(20,54,55,65) \text {. } \\
\text { Peu d'études ont examiné le } \\
\text { taux d'accès aux soins } \\
\text { médicaux chez les } \\
\text { personnes recevant un } \\
\text { diagnostic de VIH par test de } \\
\text { dépistage rapide. }\end{array}$ \\
\hline $\begin{array}{l}\text { Turner (2013) } \\
\text { (19) }\end{array}$ & $\begin{array}{l}\text { Acceptabilité globale : } 35 \% \text { à } 93 \% \text { ( } 70- \\
80) \text {. } \\
\text { Le taux d'acceptation le plus bas a été } \\
\text { constaté dans une clinique externe pour } \\
\text { adolescents }(35 \%)(73) \text {. } \\
\text { Les taux d'acceptation les plus élevés } \\
\text { dans les services d'urgence ( } 83 \% \text { et } \\
93 \%)(74,77) \text {. } \\
\text { Entre l'option de tests rapides et de tests } \\
\text { traditionnels, les jeunes sélectionnent les } \\
\text { tests rapides } 70 \% \text { du temps (73). }\end{array}$ & $\begin{array}{l}\text { Les participants ayant choisi un test } \\
\text { rapide étaient plus susceptibles de } \\
\text { recevoir leurs résultats de tests dans } \\
\text { la période de suivi, comparativement } \\
\text { aux participants qui choisissaient le } \\
\text { test traditionnel }(91,3 \% \text { c. } 46,7 \% ; \\
\text { rapport de cotes, } 12 ; \text { IC à } 95 \% 3,98 \\
-36,14)(73) \text {. } \\
100 \% \text { des jeunes âgés de } 13 \text { à } \\
17 \text { ans ayant accepté le dépistage } \\
\text { rapide reçoivent leurs résultats (77). }\end{array}$ & s.o. \\
\hline
\end{tabular}

ITS $=$ infections transmissibles sexuellement

${ }^{2} \mathrm{HARSAH}=$ hommes ayant des relations sexuelles avec d'autres hommes

\section{Rapport coût-avantage}

Sur les 17 études de modélisation examinées par Dibosa-Osadolor et al., sept études se sont penchées sur les tests diagnostiques pour détecter le VIH. Quatre études de modélisation ont évalué de façon précise les tests de dépistage rapide avec notification immédiate du patient en contexte clinique. Les auteurs ont conclu que les tests de dépistage rapide du VIH étaient plus rentables que les tests traditionnels en laboratoire avec notification immédiate du patient (21). Toutefois, la majorité des études de modélisation de tests de dépistage rapide examinées étaient basées sur des modèles statiques qui n'incluent pas de facteurs chronologiques. Cela peut potentiellement donner lieu à une surestimation de la rentabilité de maladies infectieuses $(21,22)$. Dans cet examen, aucune information n'était fournie sur les coûts directs et indirects des tests rapides ou sur le coût par année de vie ajustée par la qualité.

\section{Discussion et conclusion}

Notre étude rapide de huit examens systématiques a permis de déterminer que les tests de dépistage rapide du VIH présentent une acceptabilité généralement élevée, particulièrement chez les femmes enceintes, des écarts dans la réception des résultats de tests, et une incidence limitée sur la collaboration aux soins. Un examen a permis de déterminer que les tests de dépistage rapide étaient rentables, mais les études étaient basées sur un modèle statique et non dynamique; par conséquent, des études plus approfondies sont requises pour déterminer l'incidence des tests rapides sur la collaboration aux soins et leur rentabilité.

La méthode d'étude rapide est une approche relativement nouvelle qui présente ses forces et faiblesses. Cette méthode a comme force qu'elle permet de rapidement résumer les données pour les décideurs. En outre, les données sont présentées de façon transparente, ce qui permet aux utilisateurs de les évaluer et de prendre des décisions éclairées. 
Toutefois, quelques limites doivent être prises en considération au moment de l'examen des résultats. Le court délai du processus d'examen rapide peut faire que l'on passe à côté d'études n'ayant pas été incluses dans les examens, ce qui pose un risque de biais en raison de l'absence de renseignements pertinents. Ce court délai peut également exclure des examens systématiques récemment publiés ou qui sont en cours de publication $(23,24)$. De plus, les données de certaines études individuelles ont été citées plus d'une fois dans les examens systématiques, ce qui peut augmenter le niveau de confiance dans les résultats présentés dans cette étude rapide (24, 25). Enfin, les examens systématiques incluaient des études provenant de différents pays et donc de différents types de tests rapides de dépistage du VIH; par conséquent, les résultats de cet examen pourraient ne pas être extrapolables à d'autres tests rapides ou dans le contexte canadien.

II semble que le fait d'offrir des tests de dépistage rapide du VIH dans différents contextes soit hautement efficace lorsque les résultats des tests peuvent être obtenus rapidement. Cela suggère que ces tests rapides pourraient diminuer la proportion de personnes qui ignorent leur sérologie $\mathrm{VIH}$, et ils méritent donc d'être étudiés de façon plus approfondie. De plus amples recherches pourraient comparer l'efficacité d'une population et d'un contexte à l'autre, ainsi qu'explorer des façons d'améliorer la collaboration aux soins. II serait utile d'effectuer une étude de rentabilité basée sur un modèle dynamique.

\section{Remerciements}

Les auteurs remercient Margaret Gale-Rowe, John Kim, Lisa Pogany et Tom Wong pour leur examen du présent article et leur participation à sa rédaction. Les auteurs souhaitent également remercier Cindy Smalley et Elizabeth Dekens pour leur contribution à la recherche de la documentation.

\section{Conflit d'intérêts}

Aucun.

\section{Financement}

Cette étude a été appuyée par l'Agence de la santé publique du Canada.

\section{Références}

(1) Public Health Agency of Canada Summary: Estimates of HIV prevalence and incidence in Canada, 2011. Ottawa: PHAC; 2011. http://www.phac-aspc.gc.ca/aids-sida/publication/survreport/estimat2011-fr.php

(2) Anglemyer A, Horvath T, Rutherford G. Antiretroviral therapy for prevention of HIV transmission in HIV-discordant couples. Journal of American Medical Association. 2013; 310(15):1619.

(3) Fielden S, Lindegger M, Pederson H, McAloney C, Krajden M, Olgilvie G, et al. Evaluation findings from the pilot phase of BC's provincial point-of-care HIV testing program: The first 18 months. British Columbia: British Columbia Centre for Disease Control; 2013. http://www.bccdc.ca/NR/rdonlyres/0A26E458-1D17-4404-AB05A81DE8B69260/0/CPS_POC_Program_Eval_Report_20130823.pdf

(4) SPOT: Find out where you stand - rapid, free and anonymous HIV testing. 2009. http://www.spottestmontreal.com/En/default.aspx.

(5) Public Health Agency of Canada. Human immunodeficiency virus HIV screening and testing guide. Ottawa: PHAC; 2013. http://www.catie.ca/sites/default/files/EN_HIV-Screening-Guide-2013.pdf

(6) Public Health Agency of Canada. Point-of-care HIV testing using rapid HIV test kits: Guidance for health-care professionals. Canada Communicable Disease Report. 2007;33S2.

(7) Gaydos CA, Hsieh Y, Harvey L, Burah A, Won H, Jett-Goheen M, et al. Will patients "opt in" to perform their own rapid HIV test in the emergency department? Annals of Emergency Medicine. 2011; 58(1 SUPPL.):S74-8.

(8) Bateganya M, Abdulwadud OA, Kiene SM. Home-based HIV voluntary counselling and testing (VCT) for improving uptake of HIV testing. Cochrane database of systematic reviews (Online). 2010;7.

(9) Pant Pai N, Sharma J, Shivkumar S, Pillay S, Vadnais C, Joseph L, et al. Supervised and unsupervised self-testing for HIV in highand low-risk populations: A systematic review. PLoS Medicine. 2013; 10(4):e1001414. 
(10) Spielberg F, Branson BM, Goldbaum GM, Lockhart D, Kurth A, Rossini A, et al. Choosing HIV counseling and testing strategies for outreach settings: A randomized trial. Journal of Acquired Immune Deficiency Syndromes. 2005 Mar 1; 38(3):348-55.

(11) Pai NP, Vadnais C, Denkinger C, Engel N, Pai M. Point-of-care testing for infectious diseases: Diversity, complexity, and barriers in low- and middle-income countries. PLoS Medicine. 2012; 9(9):e1001306.

(12) Arora DR, Maheshwari M, Arora B. Rapid point-of-care testing for detection of HIV and clinical monitoring. ISRN AIDS. 2013; 287269.

(13) Khangura S, Konnyu K, Cushman R, Grimshaw J, Moher D. Evidence summaries: The evolution of a rapid review approach. Systematic Reviews. 2012; 1(10).

(14) Shea BJ, Grimshaw JM, Wells GA, Boers M, Andersson N, Hamel C, et al. Development of AMSTAR: A measurement tool to assess the methodological quality of systematic reviews. BMC Medical Research Methodology. 2007; 7(10).

(15) Pai NP, Tulsky JP, Cohan D, Colford JM, Reingold AL. Rapid point-of-care HIV testing in pregnant women: A systematic review and meta-analysis. Tropical Medicine and International Health. 2007 Feb 2007; 12(2):162-73.

(16) Roberts KJ, Grusky O, Swanson AN. Outcomes of blood and oral fluid rapid HIV testing: A literature review, 2000 -2006 | abstract. AIDS Patient Care and STDs. 2007; 21(9):621-637.

(17) Bateganya M, Abdulwadud OA, Kiene SM. Home-based HIV voluntary counseling and testing in developing countries (review). Cochrane Review. 2007; (4):1-28.

(18) Napierala Mavedzenge S, Baggaley R, Corbett EL. A review of self-testing for HIV: Research and policy priorities in a new era of HIV prevention. Clinical Infectious Diseases. 2013; 57(1):126-138.

(19) Turner SD, Anderson K, Slater M, Quigley L, Dyck M, Guiang CB. Rapid point-of-care HIV testing in youth: A systematic review. Journal of Adolescent Health. 2013; 53(6):683-391.

(20) Centers for Disease Control and Prevention (CDC). Routinely recommended HIV testing at an urgent care clinic - Atlanta, GA, 2000. Morbidity \& Mortality Weekly Report (MMWR). 2001; 50(538-541).

(21) Dibosa-Osadolor O, Roberts T. Economic evaluation, human immunodeficiency virus infection and screening: A review and critical appraisal of economic studies. International Journal of Technology Assessment Health Care. 2010; 26(03):301-308.

(22) Welte R, Postma M, Leidl R, Kretzschmar M. Costs and effects of chlamydial screening: dynamic versus static modelling. Sexually Transmitted Diseases. 2005; 32(8):474-483.

(23) Watt A, Cameron A, Sturm L, Lathlean T, Babidge W, Blamey S, et al. Rapid reviews versus full systematic reviews: An inventory of current methods and practice in health technology assessment. International Journal of Technology Assessment in Health Care. 2008; 24(2).

(24) Ganann R, Ciliska D, Thomas H. Expediting systematic reviews: Methods and implications of rapid reviews. Implementation Science. 2010; 5(56).

(25) Moher D, Tetzlaff J, Tricco AC, Sampson M, Altman DG. Epidemiology and reporting characteristics of systematic reviews. PLoS Medicine. 2007; 4(3):e78.

(26) Fylkesnes K, Siziya S. A randomized trial on acceptability of voluntary HIV counselling and testing. Tropical Medicine and International Health. 2004 May; 9(5):566-572.

(27) Wolff B, Nyanzi B, Katongole G, Ssesanga D, Ruberantwari A, Whitworth J. Evaluation of a home-based voluntary counselling and testing intervention in rural Uganda. Health Policy Plan. 2005 Mar; 20(2):109-116.

(28) Coco A. The cost-effectiveness of expanded testing for primary HIV infection. The Annals of Family Medicine. 2005; 3(5):391-399.

(29) Paltiel AD, Weinstein MC, Kimmel AD, Seage GR, Losina E, Zhang H, et al. Expanded screening for HIV in the United States An analysis of cost-effectiveness. New England Journal of Medicine. 2005; 352(6):586-595.

(30) Paltiel AD. Expanded HIV screening in the United States: Effect on clinical outcomes, HIV transmission and costs. Annals of Internal Medicine. 2006; 145(11):797.

(31) Vickerman P, Watts C, Peeling RW, Mabey D, Alary M. Modelling the cost effectiveness of rapid point of care diagnostic tests for the control of HIV and other sexually transmitted infections among female sex workers. Sexually Transmitted Infections. 2006 Oct; 82(5):403-412.

(32) Blood Products Advisory Committee (BPAC) 2005 meeting dockets. http://www.fda.gov/ohrms/dockets/ac/cber05.html\#Blood.

(33) Feasibility and acceptability of HIV self-testing among health care workers: results of a pilot programme in hospitals in Kenya. 19th International AIDS Conference, Vienna, Austria; 2010.

(34) National AIDS and STD Control Programme. Preparedness for HIVIAIDS service delivery: the 2005 Kenya health workers survey. 2006. http://www.popcouncil.org/uploads/pdfs/horizons/KenyaHealthWorkerSurvey.pdf

(35) Carballo-Diéguez A, Frasca T, Dolezal C, Balan I. Will gay and bisexually active men at high risk of infection use over-the-counter rapid HIV tests to screen sexual partners? Journal of Sex Research. 2012; 49(4):379-387.

(36) Acceptability and ease of use of home self-testing for HIV among MSM. 19th Conference on Retroviruses and Opportunistic Infections CROI; 2012.

(37) Sharma A, Sullivan PS, Khosropour CM. Willingness to take a free home HIV Test and associated factors among internet-using men who have sex with men. Journal of the International Association of Providers of AIDS Care (Chic) 2011; 10(6):357-364.

(38) Malonza IM, Richardson BA, Kreiss JK, Bwayo JJ, Stewart GC. The effect of rapid HIV-1 testing on uptake of perinatal HIV-1 interventions: a randomized clinical trial. AIDS. 2003; 3;17(1):113-118.

(39) Rajegowda BK, Das BB, Lala R, Rao S, McNeeley DF. Expedited human immunodeficiency virus testing of mothers and newborns with unknown HIV status at time of labor and delivery. Journal of Perinatal Medicine. 2005; 28(6):458-463. 
(40) Rakgoasi SD. HIV Counselling and testing of pregnant women attending antenatal clinics in Botswana, 2001. Journal of Health, Population and Nutrition. 2005; 23(1):58-68.

(41) Shankar AV, Pisal H, Patil O, Joshi A, Suryavanshi N, Shrotri A, et al. Women's acceptability and husband's support of rapid HIV testing of pregnant women in India. AIDS Care. 2003; 15(6):871-874.

(42) Webber MP, Demas P, Enriquez E, Shanker R, Oleszko W, Beatrice ST, et al. Pilot study of expedited HIV-1 testing of women in labor at an inner-city hospital in New York City. American Journal of Perinatology. 2001; 18(1):49-58.

(43) Skolnik HS, Phillips KA, Binson D, Dilley JW. Deciding where and how to be tested for HIV: What matters most? JAIDS Journal of Acquired Immune Deficiency Syndromes. 2001; 27:292-300.

(44) Pant Pai N, Bhargava M, Sharma J, Balram B, Bois C, Joseph L, et al. Will HIV self-testing be accepted by low to medium risk educated populations? A pilot cross sectional study in students of McGill University. Canadian Journal of Infectious Diseases and Medical Microbiology: 21st Annual Canadian Conference on HIVIAIDS Research. 2012; 23(SA).

(45) Belza MJ, Rosales-Statkus ME, Hoyos J, Segura P, Ferreras E, Sanchez R, et al. Supervised blood-based self-sample collection and rapid test performance: a valuable alternative to the use of saliva by HIV testing programmes with no medical or nursing staff. Sexually Transmitted Infections. 2012 Apr; 88(3):218-221.

(46) Choko AT, Desmond N, Webb EL, Chavula K, Napierala-Mavedzenge S, Gaydos CA, et al. The uptake and accuracy of oral kits for HIV self-testing in high HIV prevalence setting: A cross-sectional feasibility study in Blantyre, Malawi. PLoS ONE. 2011; 8(10):e1001102.

(47) MacPherson P, Webb EL, Choko AT, Desmond N, Chavula K, Napierala Mavedzenge S, et al. Stigmatising attitudes among people offered home-based HIV testing and counselling in Blantyre, Malawi: Construction and analysis of a Stigma Scale. PloS One. 2011; 6(10):e26814.

(48) Kalibala S, Tun W, Muraah W, Cherutich P, Oweya E, Oluoch P. "Knowing myself first": feasibility of self-testing among health workers in Kenya. 2011. http://www.popcouncil.org/uploads/pdfs/2011HIV_KenyaHWSelfTesting.pdf.

(49) Lee S, Roehler M, Miller T, Kardos K, Almeidasantos A, Gregg R. Development of an oral fluid self-test for HIV infection: evaluation in a population of unknown risk. AIDS 2012 XIX International AIDS Conference July 22-27. Washington, DC; 2012.

(50) Orasure Technologies. Final advisory committee briefing materials: Available for public release. OraQuick In-Home HIV Test. Washington D.C.: Food and Drug Administration, Blood Products Advisory Committee; 2012.

(51) Arthur GR, Ngatia G, Rachier C, Mutemi R, Odhiambo J, Gilks CF. The role for government health centers in provision of sameday voluntary HIV counseling and testing in Kenya. JAIDS Journal of Acquired Immune Deficiency Syndromes. 2005; 40(3):329335.

(52) Centers for Disease Control and Prevention (CDC). Advancing HIV prevention: New strategies for a changing epidemic - United States. Morbidity \& Mortality Weekly Report (MMWR). 2003; 52:329-332.

(53) Bulterys M, Jamieson DJ, O'Sullivan MJ, Cohen MH, et al. Rapid HIV-1 Testing During Labor: A Multicenter Study. Journal of the American Medical Association. 2004 Jul 14, 2004; 292(2):219-23.

(54) Kendrick SR, Kroc KA, Couture E, Weinstein RA. Comparison of point-of-care rapid HIV testing in three clinical venues. AIDS. $2004 ; 18(16): 2208-2210$.

(55) Kendrick SR, Kroc KA, Withum D, Rydman RJ, et al. Outcomes of offering rapid point-of-care HIV testing in a sexually transmitted disease clinic. JAIDS Journal of Acquired Immune Deficiency Syndromes. 2005 Feb 1, 2005; 38(2):142-146.

(56) Liang TS, Erbelding E, Jacob CA, Wicker H, et al. Rapid HIV Testing of Clients of a Mobile STD/HIV Clinic. AIDS Patient Care \& STDs. 2005 Apr 2005; 19(4):253-257.

(57) Nogueira SA, Lambert JS, Albuquerque AL, Rodrigues R, Reis S, Bornia R, et al. Assessment of a rapid HIV test strategy during labor: A pilot study from Rio de Janeiro, Brazil. Journal of Human Virology. 2001; 4(5):278-282.

(58) Phillips KA, Chen JL. Willingness to use instant home HIV tests: data from the California behavioral risk factor surveillance survey. American Journal of Preventive Medicine. 2003; 24(4):340-348.

(59) Pignatelli S, Simpore J, Pietra V, Ouedraogo L, Conombo G, Saleri N, et al. Factors predicting uptake of voluntary counselling and testing in a real-life setting in a mother-and-child center in Ouagadougou, Burkina Faso. Tropical Medicine \& International Health. 2006; 11(3):350-357.

(60) Shetty AK, Mhazo M, Moyo S, von Lieven A, Mateta P, Katzenstein DA, et al. The feasibility of voluntary counselling and HIV testing for pregnant women using community volunteers in Zimbabwe. International Journal of STD \& AIDS. 2005; 16(11):755-759.

(61) Smith LV, Rudy ET, Javanbakht M, Uniyal A, et al. Client satisfaction with rapid HIV testing: Comparison between an urban sexually transmitted disease clinic and a community-based testing center. AIDS Patient Care \& STDs. 2006 Oct $2006 ; 20(10): 693$.

(62) Viani RM, Araneta MRG, Ruiz-Calderon J, Hubbard P, et al. Perinatal HIV counseling and rapid testing in Tijuana, Baja California, Mexico: Seroprevalence and correlates of HIV infection. JAIDS Journal of Acquired Immune Deficiency Syndromes. 2006; 41(1):87-92.

(63) Wurcel A, Zaman T, Zhen S, Stone D. Acceptance of HIV antibody testing among inpatients and outpatients at a public health hospital: A study of rapid versus standard testing. AIDS Patient Care \& STDs. 2005; 19(8):499-505.

(64) Perez F, Mukotekwa T, Miller A, Orne-Gliemann J, Glenshaw M, Chitsike I, et al. Implementing a rural programme of prevention of mother-to-child transmission of HIV in Zimbabwe: first 18 months of experience. Tropical Medicine \& International Health. 2004; 9(7):774-783.

(65) Lubelchek R, Kroc K, Hota B, Sharief R, et al. The role of rapid vs conventional human immunodeficiency virus testing for inpatients: Effects on quality of care. Archives of Internal Medicine. 2005; 165(17):1956-60.

(66) Metcalf CA, Douglas JM, Malotte KC, Cross H, Dillon BA, Paul SM, et al. Relative efficacy of prevention counseling with rapid and standard HIV testing: A randomized controlled trial (RESPECT-2). Sexually Transmitted Diseases. 2005; 32(2):130-138. 
(67) Forsyth BWC, Barringer SR, Walls TA, Landry ML, et al. Rapid HIV testing of women in labor: Too long a delay. JAIDS Journal of Acquired Immune Deficiency Syndromes. 2004; 35(2):151-154.

(68) Morin SF, Khumalo-Sakutukwa G, Charlebois E, Routh J, Fritz K, Lane T, et al. Removing barriers to knowing HIV status: Sameday mobile HIV testing in Zimbabwe. JAIDS Journal of Acquired Immune Deficiency Syndromes. 2006; 41(2):218-224.

(69) Keenan PA, Keenan JM. Rapid HIV testing in urban outreach: A strategy for improving posttest counselling rates. AIDS Education and Prevention. 2001; 13(6):541-50.

(70) Batey S, Hogan VL, Cantor R, Hamlin CM, Ross-Davis K, Nevin C, et al. Short communication routine HIV testing in the emergency department: Assessment of patient perceptions. AIDS Research and Human Retroviruses. 2012; 28(4):352-356.

(71) Christopoulos KA, Kaplan B, Dowdy D, Haller B, Nassos P, Roemer M, et al. Testing and linkage to care outcomes for a clinicianinitiated rapid HIV testing program in an urban emergency department. AIDS Patient Care and STDs. 2011; 25(7):439-444.

(72) Freeman AE, Sattin RW, Miller KM, Dias JK, Wilde JA. Acceptance of rapid HIV screening in a southeastern emergency department. Academic Emergency Medicine. 2009; 16(11):1156-1164.

(73) Kowalczyk Mullins TL, Braverman PK, Dorn LD, Kollar LM, Kahn JA. Adolescent preferences for human immunodeficiency virus testing methods and impact of rapid tests on receipt of results. Journal of Adolescent Health. 2010; 46(2):162-168.

(74) Haines CJ, Uwazuoke K, Zussman B, Parrino T, Laguerre R, Foster J. Pediatric emergency department-based rapid HIV testing: Adolescent attitudes and preferences. Pediatric Emergency Care. 2011; 27(1):13-16.

(75) Mullins, Tanya L Kowalczyk,MD, MS, Kollar LM, MSN, Lehmann C, Kahn, Jessica A,MD, MPH. Changes in human immunodeficiency virus testing rates among urban adolescents after introduction of routine and rapid testing. Archives of Pediatric and Adolescent Medicine. 2010; 164(9):870.

(76) Kowalczyk Mullins TL, Braverman PK, Dorn LD, Kollar LM, Kahn JA. Adolescent preferences for human immunodeficiency virus testing methods and impact of rapid tests on receipt of results. Journal of Adolescent Health. 2010; 46(2):162-168.

(77) Sattin RW, Wilde JA, Freeman AE, Miller KM, Dias JK. Rapid HIV testing in a southeastern emergency department serving a semiurban-semirural adolescent and adult population. Annals of Emergency Medicine. 2011; 58(1 SUPPL.):S60-S64.

(78) Swenson RR, Hadley WS, Houck CD, Dance SK, Brown LK. Who accepts a rapid HIV antibody test? The role of race/ethnicity and HIV risk behavior among community adolescents. Journal of Adolescent Health. 2011; 48(5):527-529.

(79) Minniear TD, Gilmore B, Arnold SR, Flynn PM, Knapp KM, Gaur AH. Implementation of and barriers to routine HIV screening for adolescents. Pediatrics. 2009; 124(4):1076-1084.

(80) Payne NS, Beckwith CG, Davis M, Flanigan T, Simmons EM, Crockett K, et al. Acceptance of HIV testing among African-American college students at a historically black university in the south. Journal of the National Medical Association. 2006; 98(12):1912-1916. 